\title{
Intraventricular Bleed Secondary to Intraventricular Antibiotics: A Case Report
}

\author{
Nikhat Sultana ${ }^{1}$, K Subba Reddy², Munshi I Alam³
}

\begin{abstract}
In case of multidrug resistant CNS infection use of intraventricular antibiotics are considered which have their own undesirable effects. ${ }^{1}$ An adult male patient who presented with multidrug resistant infection secondary to procedures done to facilitate to drain cerebrospinal fluid. Secondary to intraventricular antibiotic administration patient developed an intraparenchymal bleed with intraventricular extension; as a result of the bleed there was persistently raised intracranial pressure (ICP). The harmful effects of intraventricular antibiotics have to always be considered before taking a decision to start it. Appropriate precaution and low threshold of suspicion is required to rule out complications. Keywords: CNS infection, Intraventricular antibiotics Indian Journal of Critical Care Medicine (2019): 10.5005/jp-journals-10071-23268
\end{abstract}

\section{BACKGROUND}

Intraventricular therapy of antibiotic is reserved for multidrug resistant organisms causing central nervous infection (CNS) secondary to procedures done to facilitate cerebrospinal fluid (CSF) drainage.

\section{Case Description}

A 52-year-old hypertensive male who previously underwent decompressive craniectomy for spontaneous right frontal haemorrhage followed by ventricular-peritoneal shunt (VP shunt) 10 months presented with fever and vomiting, blood investigation were within normal limit (Figs 1 and 2). CT brain plain showed postoperative communicating hydrocephalus with periventricular seepage of CSF and cerebral edema (Fig. 3). CSF culture showed pansensitive Pseudomonas. VP shunt was removed, culture showed Pseudomonas. Antibiotics ceftazidime $2 \mathrm{~g} 8$ th hourly based on the sensitivity was added.

In view of persistent drop in sensorium external ventricular drain (EVD) inserted. Persistent fever spikes for 9 days (leukocyte

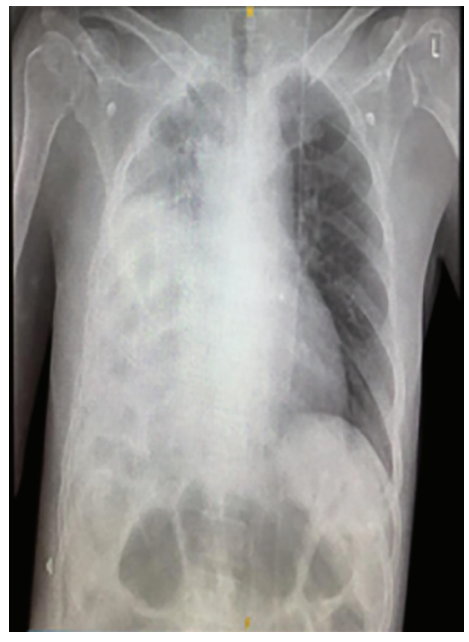

Fig 1: Chest X-ray on admission
${ }^{1}$ Department of Neuroanesthesia and Critical Care, Apollo Health City, Hyderabad, Telangana, India

2,3 Department of Neurocritical Care, Apollo Hospital, Hyderabad, Telangana, India

Corresponding Author: Nikhat Sultana, Department of Neuroanesthesia and Critical Care, Apollo Health City, Hyderabad, Telangana, India, Phone: 9036184060, e-mail: drniki1990@gmail.com

How to cite this article: Sultana N, Reddy KS, Alam MI. Intraventricular Bleed Secondary to Intraventricular Antibiotics: A Case Report. Indian J Crit Care Med 2019;23(10):484-485.

Source of support: Nil

Conflict of interest: None

count 28900 which decreased to 11100 in 2 days) Intraventricular gentamicin $5 \mathrm{mg}$ daily with $80 \mathrm{mg}$ IV thrice daily was added. Persistent fever in view of which EVD was changed. Repeat CSF culture showed multidrug resistant Pseudomonas sensitive to colistin. Started on Colistin (4.5 million IV BD along with intraventricular 1.2 lakh units OD). Repeat CSF culture showed no growth and was afebrile for

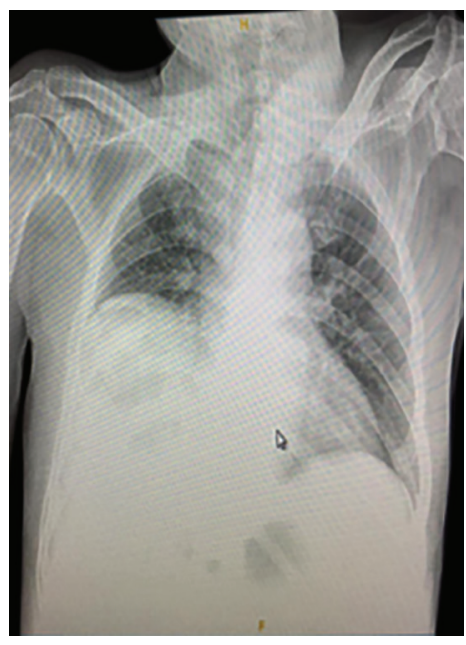

Fig 2: Chest X-ray after starting treatment

(0) The Author(s). 2019 Open Access This article is distributed under the terms of the Creative Commons Attribution 4.0 International License (https://creativecommons. org/licenses/by-nc/4.0/), which permits unrestricted use, distribution, and non-commercial reproduction in any medium, provided you give appropriate credit to the original author(s) and the source, provide a link to the Creative Commons license, and indicate if changes were made. The Creative Commons Public Domain Dedication waiver (http://creativecommons.org/publicdomain/zero/1.0/) applies to the data made available in this article, unless otherwise stated. 


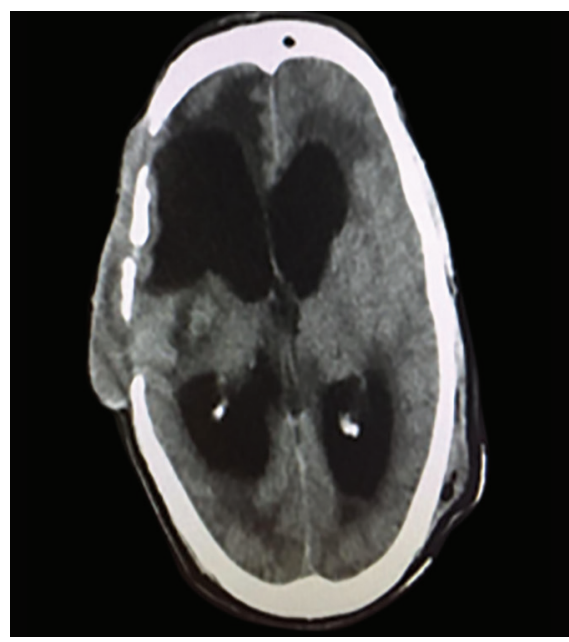

Fig. 3: Computerized tomography (CT) of brain after removal of VP shunt

next 6 days, on 7th day there was persistent hypertension followed seizure leading to acute circulatory collapse. CT brain showed intraventricular bleed in lateral ventricles, $3 \mathrm{rd}$ and 4 th ventricles, dilated lateral and third ventricle (Fig. 4). Electroencephalography (EEG) showed on going seizure activity from right hemisphere intraventricular colistin stopped. Persistent status epilepticus inspite of multiple antiepileptics.

Electroencephalography showed diffuse nonspecific electrophysiological dysfunction followed by electrical silence after few hours. Pupils were bilaterally dilated and fixed. CT brain showed increase in size of intraventricular bleed with pressure on the brainstem. Patient declared brain dead after 26 days of hospitalization

\section{Discussion}

EVDs and other CNS shunts are a mainstay in the management of hydrocephalus secondary to neurological injury; unfortunately, they may become infected. ${ }^{1,2}$ While skin flora predominates, infection with gram-negative bacilli can occur as well, possibly by introduction during surgery or via retrograde infection in the case of ventriculoperitoneal shunts. ${ }^{3}$ Ventriculitis might be caused due to infection, intrathecal injection of antibiotics. Intraventricular hemorrhage could be due to ventriculitis leading to fragile vasculature. ${ }^{4}$ There is evidence that colistin itself can cause neovascularization and these under developed vessels are fragile. ${ }^{5}$ The above factors under even a slightly stressful condition may lead

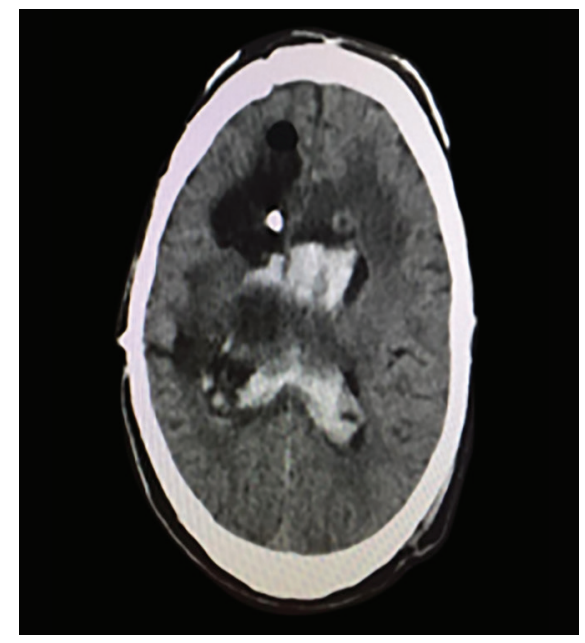

Fig. 4: CT brain showing intraventricular hemorrhage

to vascular disruption. Hence, advantage and disadvantage need to be weighed adequately.

\section{Ethical Consideration}

Waiver of consent was obtained from patient's wife.

\section{References}

1. Lee JK, Seok JY, Lee JH, Choi E, Phi JH, Kim S-K, et al. Incidence and risk factors of ventriculoperitoneal shunt infections in children: a study of 333 consecutive shunts in 6 years. J Korean Med Sci. 2012;27(12):1563-1568. doi:10.3346/jkms.2012.27.12.1563

2. Tunkel AR, Hartman BJ, Kaplan SL, Kaufman BA, Roos KL, Scheld WM, et al. Practice guidelines for the management of bacterial meningitis. Clinical Infection Disease. 2004;39(9):1267-1284. http:// dx.doi.org/10.1086/425368. Medline:15494903 Google Scholar

3. Schina M, Spyridi E, Daoudakis M, Mertzanos E, Korfias S. Successful treatment of multidrug-resistant Pseudomonas aeruginosa meningitis with intravenous and intrathecal colistin. Int J Infect Dis. 2006;10(2):178-179. http://dx.doi.org/10.1016/j.ijid.2005.03.005. Medline:16263315 Google Scholar

4. De Bonis P, Lofrese G, Scoppettuolo G, Spanu T, Cultrera R, Labonia $M$, et al. Intraventricular versus intravenous colistin for the treatment of extensively drug resistant Acinetobacter baumannii meningitis. European journal of neurology: the official journal of the European Federation of Neurological Societies. 2016;23(1):68-75. doi:10.1111/ ene.12789.

5. Klingemann HG, Egbring R, Kaffarnik H. [Effects of polymyxin B and $\mathrm{E}$ (colistin) on blood coagulation, thrombocyte function, and fibrin structure (author's transl)] Arzneimittelforschung. 1980;30(10);17191721. PMID: 6254544. 